\title{
A PROPERTY OF CONTINUITY*
}

\author{
BY D. C. GILLESPIE
}

If $\xi$ and $\eta$ are two points of the interval $(a, b)$ in which the function $f(x)$ is continuous, then the function takes on all values between $f(\xi)$ and $f(\eta)$ as $x$ changes from $\xi$ to $\eta$. This property, which we shall designate by $(A)$, is common to all continuous functions, but it is possessed also by other functions. It was shown, for example, by Darboux $\dagger$ that all derived functions possess property $(A)$, and it was pointed out by Lebesgue $\ddagger$ that still other functions which are neither continuous nor derived have the property.

The present note is concerned with functions having property $(A)$. The character of the discontinuities that such a function may have is shown. Additional conditions which are sufficient to insure that the functions be continuous or continuous and monotone follow. A theorem stating that when all the discontinuities of a function are of a certain kind it has property $(A)$ is proved, and a function having property $(A)$ and having its set of points of continuity and its set of points of discontinuity each everywhere-dense is constructed.

If $c$ is a point of discontinuity of a function $f(x)$ having property $(A)$, then in any interval about $c$ the function takes on all values between its maximum $\S$ and minimum at $c$. If $f(x)$ is unbounded, for example from above, in the neighborhood of $c$, the function could have no maximum at $c$, in which case, obviously, $f(x)$ would have, in any interval about $c$, all values greater than its minimum at $c$.

As an immediate consequence of the character of the discontinuities of a function having property $(A)$, it follows that such a function will be continuous unless the set of values it assumes an infinite number of times fills at least one interval. The converse of this statement is not true. An examination

* Presented to the Society, Sept. 7, 1920.

$\dagger$ AnNales de l'Ecole NoRmale (2), vol. 4 (1875), pp. 109, 110.

$\ddagger$ Leçons sur l'Intégration, p. 92.

$\S$ Hobson, Theory of Functions of a Real Variable, p. 234, for definition of maximum and minimum of a function at a point. 
of the Weierstrass non-differentiable continuous function reveals the fact that it takes on each value between its value at 0 and its value at 1 an infinite number of times for values of the variable in the interval $(0,1)$.

If then a function having property $(A)$ takes on no value more than once, it is continuous and hence also monotone. This idea is capable of a slight extension and then comes to an abrupt end. Since a function having property $(A)$ and taking on no value an infinite number of times is continuous, we shall state the extension for continuous functions.

If $f(x)$, continuous in $(a, b)$, assumes no value more than twice, it is possible to divide the interval into three parts in each of which $f(x)$ is monotone.

It will be convenient to consider two cases:

(1) $f(a)=f(b)$,

(2) $f(a) \neq f(b)$.

In case (1), $f(x)$ must have either its greatest or its least value at a point $\xi$ within $(a, b)$. In each of the intervals $(a, \xi)$ and $(\xi, b)$ the function takes on every value between $f(a)=f(b)$ and $f(\xi)$ one and therefore only one time, hence in each of these intervals $f(x)$ is monotone.

In case (2) assume $f(a)<f(b)$. Suppose now $f(x)$ has its least value at a point $\xi$ and its greatest value at a point $\eta$, where $\xi$ and $\eta$ are, of course, not necessarily distinct from $a$ and $b .^{*}$ It follows from the assumption $f(a)<f(b)$ that $\xi<\eta$, for if $\eta$ were less than $\xi$, the function would have the value $f(b)$, for example, once in the interval $(a, \eta)$, once in the interval $(\eta, \xi)$ and at $b$. In the interval $(a, \xi)$ the function has its least value at $\xi$ and it must have its greatest value at $a$, since, if the function were greater than or equal to $f(a)$ within $(a, \xi)$, it would assume the value $f(a)$ at least three times: once in the interval $(\xi, \eta)$, once within $(a, \xi)$ and at $a$. This function having in the interval $(a, \xi)$ its greatest value at $a$ and its least value at $\xi$, can take on no value two times in $(a, \xi)$. For suppose the function has the value $M$ at two points $p_{1}$ and $p_{2}$ within $(a, \xi)$, then, unless $f(x)=M$ has a

* If $f(x)$ have an extreme value at $a$ or $b$, take $\xi=a$ or $\eta=b$. 
root within $\left(p_{1}, p_{2}\right)$, either $f(x)>M$ for all points within $\left(p_{1}, p_{2}\right)$ and $f(x)$ has all values between $M$ and its greatest value in $\left(p_{1}, p_{2}\right)$ at least twice in the interval $\left(p_{1}, p_{2}\right)$ and once in the interval $\left(a, p_{1}\right)$; or $f(x)<M$ for all points within $\left(p_{1}, p_{2}\right)$ and $f(x)$ has all values between $M$ and its smallest value in $\left(p_{1}, p_{2}\right)$ at least twice in $\left(p_{1}, p_{2}\right)$ and once in $\left(p_{2}, \xi\right)$. The function then has each value between $f(a)$ and $f(\xi)$ one and only one time in $(a, \xi)$ and is therefore monotone. In the same way one shows that $f(x)$ is monotone in $(\xi, \eta)$ and $(\eta, b)$, the extreme values of the function for the intervals occurring at the end-points of the intervals.

If we assume that $f(x)$ takes on no value more than three times and that $f(a)=f(b)$, a similar argument will show that the interval may be divided into three parts in each of which the function is monotone.

This idea is capable of no further extension, since a continuous function may take on no value more than three times in an interval and yet be such that it is impossible to divide the interval into a finite number of parts in which the function is monotone. This statement is verified by the example:

$$
\begin{aligned}
& f(x)=\pi x+x^{2} \sin (\pi / x) \text { for } 0<x \leqq 1, \\
& f(0)=0 .
\end{aligned}
$$

The function has proper maximum points at $1 / 2,1 / 4,1 / 6, \cdots$ and proper minimum points at the roots of the equation $\tan (\pi / 2 x)=-(2 x / \pi)$, which occur in the intervals $(1 / 2,1)$, $(1 / 4,1 / 3),(1 / 6,1 / 5)$, etc. In the interval $[1 /(2 n+1)$, $1 /(2 n-1)]$ the function has one maximum point and one minimum point, hence takes on no value more than three times in this interval. Moreover,

for

$$
\frac{\pi}{2 n+1} \leqq f(x) \leqq \frac{\pi}{2 n-1}
$$

$$
\begin{gathered}
\frac{1}{2 n+1} \leqq x \leqq \frac{1}{2 n-1}, \\
f(x)>\frac{\pi}{2 n-1} \quad \text { for } \quad x>\frac{1}{2 n-1}
\end{gathered}
$$


and

$$
f(x)<\frac{\pi}{2 n+1} \quad \text { for } \quad x<\frac{1}{2 n+1} .
$$

We have shown that a function having property $(A)$ assumes in any interval about a point of discontinuity every value between its maximum and minimum at this point. The converse of this is not true; for example, $f(x)=1+x+\sin (\pi / x)$ for $0<x \leqq 1, f(0)=1$ and $f(x)=x$ for $-1 \leqq x<0$ has at zero its only discontinuity. In every interval about zero it assumes every value between 0 , its minimum at 0 , and 2 , its maximum at 0 , and although $f(-1)=-1$ and $f(1)=2$, $f(x)=0$ has no root in $(-1,1)$. It is to be observed that the values between 0 and 2 are not assumed by the function in any interval whose upper end-point is 0 .

THEOREM. If a function $f(x)$ at every point of discontinuity $c$ takes on all values between its maximum and minimum at $c$ in every interval of which $c$ is an end-point, then the function has property $(A)$.

Suppose $\xi_{1}$ and $\eta_{1}, \xi_{1}<\eta_{1}$, are two points of the interval $(a, b)$ in which $f(x)$ is defined, and that $f\left(\xi_{1}\right)<M<f\left(\eta_{1}\right)$; we shall show that $f(x)=M$ has a root between $\xi_{1}$ and $\eta_{1}$. Consider the set of points in $\left(\xi_{1}, \eta_{1}\right)$ at which $f(x)>M$ and take the lower limit $p_{1}$ of this set. If $p_{1}=\xi_{1}$, then, since $\xi_{1}$ is the lower limit of a set of points at which $f(x)>M$, the maximum of $f(x)$ at $\xi_{1}$ is not less than $M$. If this maximum of $f(x)$ at $\xi_{1}$ is greater than $M$, then $f(x)=M$ has a root in every interval of which $\xi_{1}$ is an end-point. If $p_{1}>\xi_{1}$, then there is no point within $\left(\xi_{1}, p_{1}\right)$ for which $f(x)>M$, hence the maximum of $f(x)$ at $p_{1}$ is $M$. In any case, whether $p_{1}$ is equal to $\xi_{1}$ or greater than $\xi_{1}$, the equation $f(x)=M$ either has a root in $\left(\xi_{1}, \eta_{1}\right)$ or $f\left(p_{1}\right)<M$ and the maximum of $f(x)$ at $p_{1}$ is $M$. Suppose $M-f\left(p_{1}\right)=\epsilon$; now choose a positive $\delta<\left[\left(\eta_{1}-p_{1}\right) / 2\right]$ and such that $f(x)<M+\epsilon / 2$ in the interval $\left(p_{1}, p_{1}+\delta\right)$. Within this interval there is a point $\eta_{2}$ at which $f\left(\eta_{2}\right)>M$. Now it may be established likewise that either $f(x)=M$ has a root in $\left(p_{1}, \eta_{2}\right)$ or there exists at the 
upper end of $\left(p_{1}, \eta_{2}\right)$ a subinterval $\left(\xi_{2}, \eta_{2}\right)$ such that $f\left(\xi_{2}\right)<M$ and $f(x)>M-(\epsilon / 2)$. Thus in the interval $\left(\xi_{2}, \eta_{2}\right)$

$$
|f(x)-M|<\epsilon / 2 \text {. }
$$

Continuing this process, we obtain a sequence of intervals $\left(\xi_{n}, \eta_{n}\right)$ each lying within the preceding one and of length less than $\left(\eta_{1}-\xi_{1}\right) / 2^{n-1}$ for which $f\left(\xi_{n}\right)<M, f\left(\eta_{n}\right)>M$, $|f(x)-M|<\epsilon / 2^{n-1}$ for $x$ in $\left(\xi_{n}, \eta_{n}\right)$. Hence at the one point $p$ common to this set of intervals $f(p)=M$.

It will require only obvious changes in the preceding argument to prove a more general theorem*. Using the four symbols $\bar{f}(c+0), f(c+0), \bar{f}(c-0)$ and $f(c-0)$ to designate the upper and lower limits $\dagger$ of $f(x)$ to the right and left of $c$ respectively, this theorem may be stated:

If at every point of discontinuity $c$ of $f(x), f(c+o) \leqq f(c)$ $\leqq \bar{f}(c+0), f(c-0) \leqq f(c) \leqq \bar{f}(c-0)$ and $f(\bar{x})$ takes on every value between $f(c+0)$ and $\bar{f}(c+0)$ in every interval whose left end-point is $c$ and every value between $f(c-0)$ and $\vec{f}(c-0)$ in every interval whose right end-point is $\bar{c}$, then $f(x)$ has property (A).

The conditions thus imposed upon $f(x)$ are not only sufficient but also clearly necessary for the existence of property $(A)$.

As illustrations of functions having property $(A)$ we have on the one hand continuous function or certain functions with a finite number of discontinuities, and on the other hand totally discontinuous functions. $\ddagger$

The following function $\S$ defined by a uniformly convergent series has property $(A)$ and has its set of points of continuity and its set of points of discontinuity each everywhere-dense:\|

$$
f(x) \|=\sum_{n=1}^{\infty} \frac{1}{n^{2}} g\left(x-r_{n}\right),
$$

* E. R. Hedrick pointed out to me that the argument really proved this more general theorem.

$\dagger$ Hobson, loc. cit., p. 231.

$\ddagger$ Lebesgue, loc. cit., p. 90.

§ Pierpont, Theory of Functions of a Real Variable, Vol. II, p. 463, Ex. 1.

\| W. A. Hurwitz suggested the probability of the function defined possessing the properties enumerated. The present note grew out of a study of this example. 
where $r_{1}, r_{2}, r_{3}, \cdots$, denotes the set of rational numbers, $g(x)=\sin (\pi / x)$ for $x=0$ and $g(0)=0$.

The function $f(x)$ is continuous at all irrational points and has a discontinuity of the second kind with oscillation equal to $2 / n^{2}$ at each rational point $r_{n}$. To prove the function has property $(A)$, it is sufficient, in consequence of the theorem just established, to show that $f(x)=M$, where $f\left(r_{n}\right)-\left(1 / n^{2}\right)$ $<M<f\left(r_{n}\right)+1 / n^{2}$, has a root in every interval of which $r_{n}$ is an end-point. From the definition of $f(x)$ it is obvious that corresponding to any positive number $\epsilon$ there exists a positive number $\delta$ such that the oscillation of $f(x)$ at any point lying inside the interval $\left(r_{n}, r_{n}+\delta\right)$ is less than $\epsilon$. Within $\left(r_{n}, r_{n}+\delta\right)$ there is a point $\eta_{1}$ at which $f\left(\eta_{1}\right)>M$ and then within $\left(r_{n}, \eta_{1}\right)$ a point $\xi_{1}$ at which $f\left(\xi_{1}\right)<M$. By taking the upper limit of those points in $\left(\xi_{1}, \eta_{1}\right)$ at which $f(x)<M$ and proceeding as before, a convergent sequence of intervals $\left(\xi_{m}, \eta_{m}\right)$ where $f\left(\xi_{m}\right)<M<f\left(\eta_{m}\right)$ and for which the oscillation at each point is less than $\epsilon / 2^{m}$ may be constructed. At the one point $p$ common to the set of intervals, $f(p)=M$. The same is true, of course, of intervals bounded above by $r_{n}$.

One could infer, from facts already established, the existence of a function having property $(A)$ and having its set of points of continuity and its set of points of discontinuity each everywhere-dense. For functions which are everywhere oscillating and which nevertheless have a derivative at every point of an interval have been constructed.* The derived function has, of course, property $(A)$; this derived function being the limit of a sequence of continuous functions has its points of continuity everywhere-dense; $\dagger$ its points of discontinuity are also everywhere-dense since it is both positive and negative in every interval.

Cornell University

* Köpcke, Mathematische Annalen, vols. 34 and 35.

† Baire, Legons sur les Foncions Discontinues, p. 98. 